\title{
Rat dorsal prostate is necessary for vaginal adhesion of the seminal plug and sperm motility in the uterine horns
}

\author{
José L Tlachi-López' ${ }^{1}$ Aurora López ${ }^{1}$, Kurt Hoffman², Javier Velázquez-Moctezuma³, Mario García-Lorenzana³ \\ and Rosa Angélica Lucio $^{*}$ \\ ${ }^{1}$ Centro Tlaxcala de Biología de la Conducta, Universidad Autónoma de Tlaxcala, Carretera Tlaxcala-Puebla km 1.5 s/n, Loma Xicotencatl 90062 , Tlaxcala, México. \\ ${ }^{2}$ Centro de Investigación en Reproducción Animal. Universidad Autónoma de Tlaxcala-CINVESTAV, Carretera San Martín Texmelucan-Tlaxcala km 10.5 s/n, San Felipe \\ Ixtacuixtla 90120, Tlaxcala, México. \\ 3 Área de Neurociencias, Universidad Autónoma Metropolitana-Iztapalapa, Av. Michoacán y la Purísima 38340 Iztapalapa 55535. México, DF, México.
}

\begin{abstract}
The rat prostate comprises dorsal, ventral and lateral lobes that are morphologically and biochemically distinct. Lesions to these structures are expected to affect the quality of the ejaculate and male fertility. In experiment 1, we analyzed ejaculate parameters of males that had chemical lesions of the dorsal or ventral lobes. At pre-lesion and at 5 and 20 days post-lesion males were mated, and after ejaculation, seminal fluid and seminal plug were obtained from the mated females. In experiment 2, the ventral lobes were ablated, and the ejaculate was analyzed. In experiment 3, the fertility of males with chemically-lesioned dorsal lobes or ablation of the ventral lobes was evaluated. Chemical lesion of the dorsal lobe prevented the adhesion of the seminal plug to vaginal walls. When these males were tested at 5-days postlesion, no sperm were found in uterus, and at 20-days post-lesion, the few sperm encountered showed slow progressive motility. None of the females that mated with dorsal lobe-lesioned males became pregnant. However, chemical lesion or ablation of the ventral lobes did not affect ejaculate or fertility. Our results indicate that the dorsal prostatic lobes are indispensable for reproductive success in males, and define parameters of ejaculate with which fertility can be estimated.
\end{abstract}

Key terms: ejaculation, characteristics of semen, copulatory plug, prostate, seminal analysis, sperm number.

\section{INTRODUCTION}

In the rat, secretions of the accessory sexual glands and spermatozoa are deposited into the vagina during ejaculation. Immediately after, seminal fluids coagulate to form the copulatory plug (Matthews Jr and Adler, 1978). This seminal plug facilitates transcervical sperm transport (Blandau, 1945; Matthews and Adler, 1977; Matthews Jr and Adler, 1978). Approximately $1 \%$ of the ejaculate contains spermatozoa; the rest corresponds to secretions of the accessory sexual glands, which include the bulbourethral and coagulating glands, seminal vesicles and prostate (Setchell et al., 1994). These sex glands differ among males of different species with respect to number and shape, but also some of these sex glands are absent. Nevertheless, the prostate is present in all male mammals (Luke and Coffey, 1994).

The rat prostate is a large gland comprising dorsal, ventral and lateral portions, with each having a right and left lobe (Hayashi et al., 1991; Jesik et al., 1982). The dorsal lobes are located inferior and posterior to the urinary bladder, below and behind the attachment of the seminal vesicles and coagulating glands (Hayashi et al., 1991). The ventral lobes are found on the ventral aspect of the urethra immediately below the urinary bladder. The lateral lobes lie just below the seminal vesicles and coagulating glands, partially overlapping the ventral lobes and, dorsally, blend with the dorsal lobes. Morphological analysis by micro-dissection reveals that all lobes are composed of ducts that emerge from the urethra and arborize distally. The dorsal lobes have 5-6 pairs of main ducts with true acinar termini (Hayashi et al., 1991). Each ventral lobe has 2-3 slender main ducts, whereas each lateral lobe has
5-7 long main ducts and 5-6 short main ducts. The rat prostate secretes a variety of substances that enter the urethra to form the prostatic portion of the semen. The secretion products are biochemically heterogeneous according to their lobe of origin. The dorsal lobes secrete dorsal-protein I and dorsalprotein II, among other products (Seitz et al., 1990), and are the major sites of fructose secretion (Humphrey and Mann, 1949; Mann, 1964). Ventral lobe secretions include citrate, spermine and spermidine (Price and William-Ashman, 1961), aminopeptidases (Vanha-Perttula and Jauhiainen, 1983), and plasminogen activator (Wilson et al., 1988). Finally, lateral lobes are the major zinc-secreting portion of the prostate (Gunn and Gould, 1957).

These lobe-specific secretions most likely contribute significantly to reproductive success. Therefore, the aim of the present work was to determine the characteristics of the ejaculate obtained from males having dorsal or ventral prostatic lobe lesions, and evaluate the fertility of these males.

\section{MATERIALS AND METHODS}

In pilot studies, attempts were made to extirpate the dorsal prostatic lobes. We encountered two difficulties: first, the surgical manipulation required to remove these lobes damaged adjacent pelvic structures, particularly the base of the urinary bladder and the urethral dorsal wall. For this reason, we did not ablate the dorsal lobes in the present study; instead, we lesioned them chemically. Second, surgical removal of the lateral lobes eliminates the autonomic innervation of the penile erectile tissue and other reproductive organs. This innervation arises from the major pelvic ganglia, which are attached to the 
lateral lobes of the prostate (Hebel and Stromberg, 1986; Dail et al., 1989). Ablation of these lobes abolishes penile erection, thereby preventing the male from intromitting and ejaculating. For this reason, ablation of the lateral lobes was not attempted. Likewise, chemical lesioning was not attempted because, due to the transparency of the sclerosing agent, it was not possible to determine the extent of its diffusion within the tissue. Thus, the present experimental procedures included chemically lesioning the dorsal lobes, and chemical lesion or surgical ablation of the ventral ones.

\section{Animals}

Adult male (300-450 $\mathrm{g}$ of body weight and female (200-300 $\mathrm{g}$ of body weight) Wistar were used rats in this study, obtained from the vivarium of the Tlaxcala Center for Behavioral Biology. Rats were kept under standard vivarium conditions, in a room with controlled temperature and under an inverted 12/12 light-dark cycle, with food (Purina Chow) and water available ad libitum. All procedures described in this study were in accordance with the guidelines of the Laws and Codes of Mexico in Article Seven of the Regulations of the General Law of Health Regarding Health Research, and followed the guidelines of the NIH for the use of animals (Laws and Codes of Mexico, 1995).

Experiment 1. Chemical lesion of dorsal and ventral prostatic lobes and analysis of ejaculate:

\section{Chemical lesion procedure}

Under deep sodium pentobarbital anesthesia (Pfizer; $26 \mathrm{mg} /$ $\mathrm{kg}$; intraperitoneal) male rats were submitted to a surgical manipulation by which the prostatic lobes were exposed. Prostatic lobes were injected with $10 \mu \mathrm{l}$ of a $2 \%$ solution of tetradecil sodium sulphate (Aldrich 293938-1G) using a
Hamilton syringe (dorsal lobes, $\mathrm{n}=6$ males; ventral lobes, $\mathrm{n}=6$ males). Tetradecyl sodium sulphate is a sclerosing agent, used successfully in clinical studies, which promotes an increase in fibrotic processes, thereby resulting in drastic and irreversible impairment of tissue function (Griffin et al., 1986). This solution was injected into five different sites of each lobe $(2 \mu \mathrm{l} /$ site; Fig. 1). After each administration, the needle remained in place for one minute to prevent the solution from escaping. Animals recovered for 5 days. Previous observations in our laboratory had indicated that with this procedure and these doses, the prostatic lobes showed a clear fibrotic response.

\section{Collection and macroscopic and microscopic evaluation of ejaculate}

Sexually experienced males and ovariectomized virgin females were used. Females were brought into estrous by sequential treatment with $10 \mu \mathrm{g}$ of estradiol benzoate (SigmaAldrich E-8515) and $2 \mathrm{mg}$ of progesterone (Sigma-Aldrich P-0130), administered by subcutaneous injection $44 \mathrm{~h}$ and 4 $\mathrm{h}$, respectively, before the copulatory encounters. Males were placed into a plexiglass cylinder $(50 \mathrm{~cm}$ diameter $/ 50 \mathrm{~cm}$ high with wood shavings on the floor. Each male was tested three times: before performing the chemical lesion and at 5 and 20 days post-lesion. Each test consisted of one ejaculatory series registering the copulatory parameters (mount latency, intromission latency, ejaculation latency, number of mounts and number of intromissions). Once the male ejaculated, the female was immediately transferred from the arena to an empty cage where she was left quiet for 5 min before being anesthetized with pentobarbital $(26 \mathrm{mg} / \mathrm{kg}$; intraperitoneal; Pfizer; DF, México). After an abdominal incision, their uterine horns were tied proximally and distally, removed from the abdominal cavity, and immersed in a Petri dish containing saline solution $(0.9 \%)$ at $37^{\circ} \mathrm{C}$. This is the easiest way to eliminate blood, fat tissue and external uterine vessels, but also serves to maintain seminal fluid at a stable temperature.
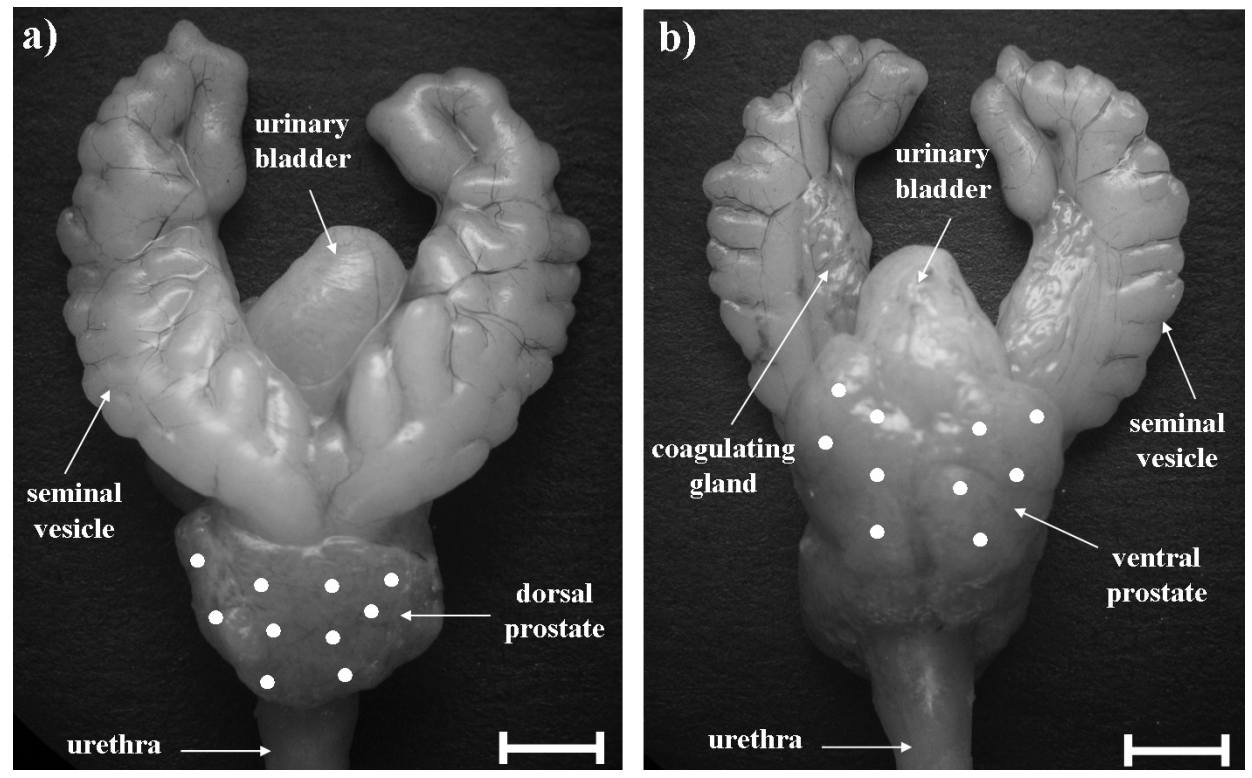

Figure 1: a) Dorsal, b) ventral views of the prostatic lobes, showing the injection sites $(\bullet=$ injection site); Bar=1 cm. 
Seminal content of both uterine horns was placed in a 1.5 $\mathrm{ml}$ micro-centrifuge tube, and maintained in a thermo-bath at $37^{\circ} \mathrm{C}$. The samples of ejaculate collected in this manner were used to evaluate the following parameters of the semen

1) Color, distinguished as off-white or transparent.

2) Viscosity, measured by the length (in $\mathrm{mm}$ ) of a thread formed after introduction and withdrawal of a tip of a transfer pipette into the semen.

3) $\mathrm{pH}$, measured placing a drop of semen on a $\mathrm{pH}$ indicator paper.

4) Sperm motility, individual motility of spermatozoa. We classified motility using a 4-category scale, according to the time in seconds the spermatozoa took to cross a horizontal line running across the observed field (the line was drawn into one of the microscope's objectives). Thus, spermatozoa whose heads were in the proximity of the horizontal line were designated to one of four categories: rapid progressive (those taking 2-3 sec to cross the line); slow progressive (those taking more than $4 \mathrm{sec}$ ); and in situ (those with non-progressive motility, generally circular or local motility); and immobile (no movement). The reading was from left to right using the 20x microscope objective, and counting 100 spermatozoa. The obtained value was expressed in percentage. The sperm motility of each sample was filmed in order to confirm the obtained results.

5) Sperm viability, nigrosin-eosin and sodium citrate stains were used to distinguish live (unstained) or dead (stained) sperm. 100 sperm were counted using the 100x microscope objective. This parameter was also expressed in percentage.

6) Sperm morphology, nigrosin-eosin and sodium citrate stains were used to distinguish normal (having a sickleshape head and large flagella) or abnormal (those with double head and fragmented or zig-zag flagella) sperm. 100 sperm were counted using the 100x microscope objective. Sperm morphology was expressed in percentage.

7) Sperm concentration, semen suctioned by a Shali pipette (diluted with $2 \%$ formaldehyde and shaken until homogenized semen was obtained) was placed in a Neubauer hemocytometer, where the sperm were counted under a 20x microscope objective. The number of spermatozoa was expressed in millions per $\mathrm{ml}$ (Lucio et al., 2009).

The seminal plug was removed from the vagina by separating the pubic symphysis and cutting the dorsal vaginal wall longitudinally. Using a spatula, short movements were performed to detach the seminal plug from lateral and ventral vaginal walls and from cervix, before carefully removing it. We recorded the following parameters:

1) Consistency, seminal plug solidity, qualitative parameter determined by direct tactile inspection.

2) Weight, seminal plug mass, expressed in mg.

3) Size, length and width of the seminal plug, expressed in $\mathrm{mm}$.

4) Volume, mass occupied by the seminal plug, expressed in $\mathrm{mm}^{3}$.

5) Cytological elements including number of single heads, single flagella and complete spermatozoa, expressed in percentage. For this, the seminal plug was cut transversely at the proximal end (approximately $1 \mathrm{~mm}$ from the surface that had been attached to the cervix), at the distal end (approximately $1 \mathrm{~mm}$ from the surface that had been at the vaginal orifice), and at the equatorial region (half way between the prior two cuts). The cut surface of each of these three sections was slid horizontally from left to right over a clean microscope slide, "drawing" four parallel lines, while taking care not to overlap them (Lucio et al., 2009). After collecting the semen and the seminal plug, the females were sacrificed.

\section{Statistical analysis}

Copulatory parameters were quantified according to methods described in previous studies (Meisel and Sachs 1994), and the quantitative data were analyzed using a Friedman test. Data from the assessment of seminal content and seminal plug were analyzed using a Friedman test. Results obtained before lesion were compared with those obtained at day 5 and 20 postlesion. Significance level was set at 0.05 (Zar, 1999).

\section{Evaluation of chemical lesion}

After the ejaculate analysis on day 20 post-lesion, all males were injected intraperitoneally with an overdose of sodium pentobarbital (Pfizer; DF, México). Additional males were lesioned (or not, in the case of 4 control males) in their dorsal $(n=4)$ or ventral $(n=4)$ prostatic lobes, and then sacrificed on day 5 post-lesion, corresponding to the first day of ejaculate analysis. In this way, we obtained a histological view of intact and chemically-lesioned prostates on days 5 and 20 postlesion.

Histological analysis was done by dissecting and removing the prostatic lobes, cutting sagitally. Tissue was fixed with bouin Duboscq (Humason, 1972), rinsed with $70 \%$ ethanol, dehydrated with $80 \%, 96 \%, 100 \%$ ethanol and cleared in a $1: 1$ mix of $100 \%$ ethanol-xylene and pure xylene. Inclusion was performed with Paraplast (Oxford Labware). 5-micrometer serial sections were obtained using a microtome (Leica RM 2135). Prostatic sections were stained using hematoxylin-eosin (Presnell and Schreibman, 1997). The sections were analyzed under a clear field light microscope (Axioskope II, Carl Zeiss).

Experiment 2. Ablation of ventral prostatic lobes and analysis of ejaculate

Surgical lesion procedure

Under deep sodium pentobarbital anesthesia (Pfizer; 26 $\mathrm{mg} / \mathrm{kg}$; intraperitoneal) and in a supine position, a midline abdominal incision was made in male rats $(n=6)$. After the identification of the urinary bladder, ventral lobes of the prostate were carefully dissected and excised. Abdominal musculature and abdominal skin were sutured and the animals were placed into individual cages to recover. At 5 and 20 days post-lesion, semen and seminal plug samples were obtained and evaluated, and statistical analysis of the data was done as described for experiment 1 .

Experiment 3. Assessment of the fertility of males with chemical lesions of the dorsal lobes or surgical ablation of the ventral lobes

\section{Chemical lesion and surgical ablation}

The dorsal prostatic lobes of 7 males were chemically lesioned and the ventral prostatic lobes of a separate group of 7 males were surgically ablated, as described above. 


\section{Assessment of fertility}

Before the chemical or surgical lesion and again at 20 days post-lesion, reproductive units were constituted (Manzo et al., 2000; Lucio et al. 2001). Each male was placed in a cage with three intact adult females during a test period of 15 days, after which the females were transferred to individual cages. The date of parturition, if one occurred, was recorded in order to determine the exact date of insemination. For analysis, the test period (15 days) was divided into 3 intervals: days 1-5, 6-10, and 11-15. The number of pregnant females within each of the three periods was determined, and the number of nonpregnant females was also recorded. Treatment groups were compared with respect to the number of pregnant females within each of the three periods. Data of fertility were analyzed using a $G$ test (Zar, 1999).

\section{RESULTS}

Experiment 1. Effect of chemical lesion of dorsal and ventral prostatic lobes

Statistical analysis did not show behavioral alterations in males with chemically lesioned dorsal or ventral prostatic lobes (data not shown).

\section{Analysis of seminal fluid}

Five days after chemically lesioning the dorsal prostatic lobe, none of the semen samples obtained from the uterine horns were off-white, due to the absence of sperm. In these cases, therefore, the evaluated viscosity corresponds only to the uterine fluid, explaining why the obtained value was zero. By contrast, at 20 days post-lesion, no alterations were observed in macroscopic parameters (color, viscosity or $\mathrm{pH}$; Table I).

The microscopic analysis could not be performed at 5 days post-lesion due to the absence of spermatozoa in the uterus; therefore, the sperm concentration was zero. At 20 days post-lesion, the negative effect continued because we observed a significant reduction in sperm concentration as well as a significant decrease in the percentage of spermatozoa with rapid progressive motility, compared to pre-lesion data. Interestingly, a high percentage of spermatozoa in the uterus exhibited in situ motility compared to before the lesion. Sperm viability and sperm morphology at 20 days post-lesion were similar to that observed before the lesion (Table I).

In contrast to the marked effects of the dorsal lobe lesions, chemically lesioning the ventral prostatic lobes did not significantly affect any seminal fluid parameters. Thus, prelesion data of this group of males were similar to that shown in Table I, and no changes in any parameter of seminal fluid were observed at either 5 or 20 days post-lesion (data not shown).

Analysis of the seminal plug

In the case of dorsal-lobe lesioned males, when the seminal plug was obtained, the perivaginal region was observed to be wet. In many cases, leakage of seminal fluid from the vagina was easily observed (Fig. 2a). Removal of seminal plug from the vagina was easy because it was not attached to vaginal walls and it was wet from seminal fluid. Thus, the chemical lesion of the dorsal lobes prevented the adhesion of the seminal plug to the vaginal walls and cervix. Surprisingly, the macroscopic parameters (consistency, weight, size and volume), showed no significant changes at either 5 or 20 days after the lesion, compared to pre-lesion (Table II).

TABLE I

Seminal fluid macroscopic and microscopic values before and after chemical lesion of the dorsal prostatic lobes $(n=6)$

\begin{tabular}{|c|c|c|c|c|}
\hline & \multirow{2}{*}{ Seminal fluid Parameters } & \multirow{2}{*}{ Before lesion } & 5 days & 20 days \\
\hline & & & \multicolumn{2}{|c|}{ After lesion } \\
\hline \multirow{3}{*}{ 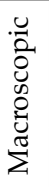 } & 1) Off-white (\% samples) & 100a & $\mathrm{Ob}$ & 100 \\
\hline & 2) Viscosity (mm) & $2 \mathrm{a}$ & $0 \mathrm{~b}$ & 2.5 \\
\hline & 3) $\mathrm{pH}$ & 8 & 8 & 8.25 \\
\hline \multirow{8}{*}{ 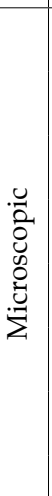 } & 4) Sperm motility sperm rapid progressive (\% spz) & $53^{\mathrm{a}}$ & sperm absence & $13.5^{\mathrm{b}}$ \\
\hline & slow progressive $(\% \mathrm{spz})$ & $24^{\mathrm{a}}$ & sperm absence & $32.25^{\mathrm{b}}$ \\
\hline & in situ $(\% \mathrm{spz})$ & $15.5^{\mathrm{a}}$ & sperm absence & $41^{\mathrm{b}}$ \\
\hline & immotility (\% spz) & $7.5^{\mathrm{a}}$ & sperm absence & $13^{\mathrm{b}}$ \\
\hline & motility rate $(0-1)$ & $0.7^{\mathrm{a}}$ & sperm absence & $0.4^{\mathrm{b}}$ \\
\hline & 5) Sperm viability (\% spz) & 71 & sperm absence & 71 \\
\hline & 6) Sperm morphology (\% spz) & 100 & sperm absence & 99.5 \\
\hline & 7) Sperm concentration $\left(10^{6} / \mathrm{ml}\right)$ & $13^{\mathrm{a}}$ & $0^{\mathrm{b}}$ & $7.5^{\mathrm{b}}$ \\
\hline
\end{tabular}

Data of parameters were expressed as median. spz=spermatozoa. Friedman test, ${ }^{\mathrm{b}} P<0.05$. 
Microscopic parameters of the seminal plug were altered only in the proximal end, and only at 5 days after the lesion; these parameters were unaltered compared to control at 20 days post-lesion. Thus, the smear of the proximal end of the seminal plug at 5 days post-lesion revealed millions of agglutinated spermatozoa (for this reason they could not be quantified; Fig. 2b), many of them displaying in situ motility. By contrast, in intact males, the smear of this end of the seminal plug normally showed single heads, single flagella (typically having a greater percentage of heads than flagella), and few complete spermatozoa (Fig. 2c). The smear of the equatorial and distal portions of the seminal plug of lesioned males showed no differences when compared with smears from these same males obtained before lesion, either at 5 or 20 days post-lesion.

Chemically lesioning the ventral prostatic lobes did not affect any of the seminal plug parameters. Thus, pre-lesion data of this group of males were similar to that shown in Table II, and no changes in any parameter of the seminal plug were observed at either 5 or 20 days post-lesion (data not shown).
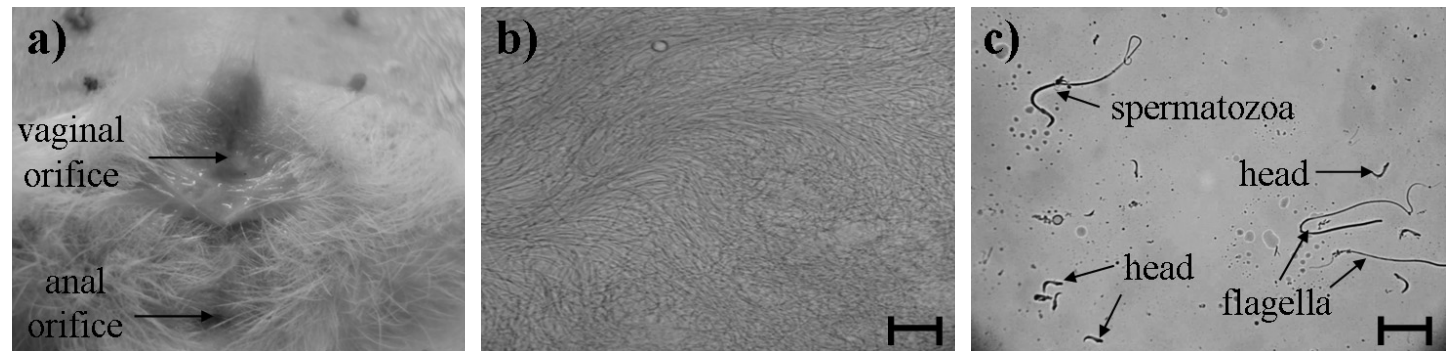

Figure 2: a) Photograph of the vaginal orifice of a female inseminated by a male with chemically-lesioned dorsal prostatic lobes. Leakage of seminal fluid can be observed. b) Photomicrograph of a smear from the proximal portion of the seminal plug from a lesioned male. Millions of spermatozoa can be observed. c) Photomicrograph of a smear of the proximal portion of the seminal plug from an intact male. Single heads, single flagella, and spermatozoa can be observed. (Bar=200 $\mu \mathrm{m})$.

TABLE II

Seminal plug macroscopic and microscopic values before and after chemical lesion of the dorsal prostatic lobes $(n=6)$

\begin{tabular}{|c|c|c|c|c|}
\hline & \multirow{2}{*}{ Seminal fluid Parameters } & \multirow{2}{*}{ Before lesion } & 5 days & 20 days \\
\hline & & & \multicolumn{2}{|c|}{ After lesion } \\
\hline \multirow{5}{*}{ 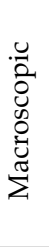 } & 1) Hardened consistency ( $\%$ of seminal plugs) & 100 & 100 & 100 \\
\hline & 2) Weight (mg) & 112 & 109 & 111 \\
\hline & 3) Size length (mm) & 13.1 & 12.1 & 12.6 \\
\hline & width $(\mathrm{mm})$ & 5.4 & 5.4 & 5.4 \\
\hline & 4) Volume $\left(\mathrm{mm}^{3}\right)$ & 100 & 98.99 & 98.35 \\
\hline \multirow{13}{*}{ 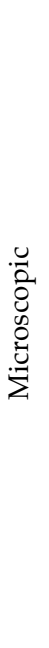 } & 5) Cytologic elements & $53^{a}$ & sperm absence & $13.5^{\mathrm{b}}$ \\
\hline & proximal portion & & & \\
\hline & single heads $(\%)$ & 51.11 & undistinguishable & 54.31 \\
\hline & single flagella $(\%)$ & 37.43 & undistinguishable & 32.92 \\
\hline & spermatozoa $(\%)$ & 11.44 & millions & 12.76 \\
\hline & equatorial portion & & & \\
\hline & single heads $(\%)$ & 69.68 & 60.26 & 86.89 \\
\hline & single flagella $(\%)$ & 17.27 & 19.19 & 11.61 \\
\hline & spermatozoa $(\%)$ & 13.03 & 20.53 & 1.49 \\
\hline & distal portion & & & \\
\hline & single heads $(\%)$ & 81.79 & 47.92 & 88.31 \\
\hline & single flagella $(\%)$ & 16.41 & 18.72 & 11.68 \\
\hline & spermatozoa $(\%)$ & 1.79 & 33.34 & 0 \\
\hline
\end{tabular}

Data of parameters were expressed as median. Friedman test, ${ }^{b} P<0.05$. 
Figure 3a; b; c shows the histological changes apparent as a result of the dorsal prostatic lobe lesion. At day 5 post-lesion, the dorsal lobes showed a massive infiltration of leukocytes in both the stroma and in the acini. When the histological examination was done 20 days post-lesion, the infiltration of leukocytes was diminished. Figure $\mathbf{3 d}$; $\mathbf{e} ; \mathbf{f}$ shows the tissue organization of the ventral prostatic lobe. The administration of the sclerosing solution induced only minor damage to this tissue. The microscopic analysis of the prostatic gland at day 5 after the chemical lesion showed a significant presence of macrophages and sparse leukocytes (neutrophyles) in the stroma, suggesting an inflammatory process. This inflammatory process was diminished when the tissue was analyzed 20 days after the lesion.

DORSAL LOBE

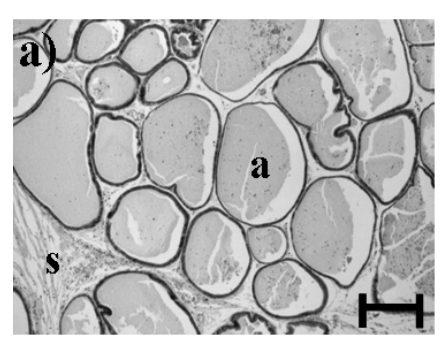

VENTRAL LOBE

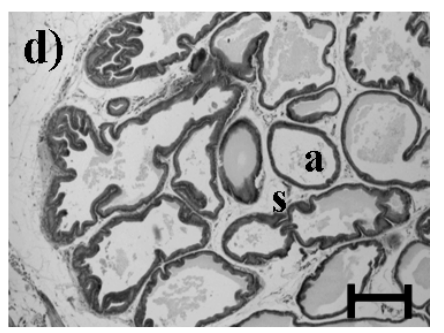

Intact

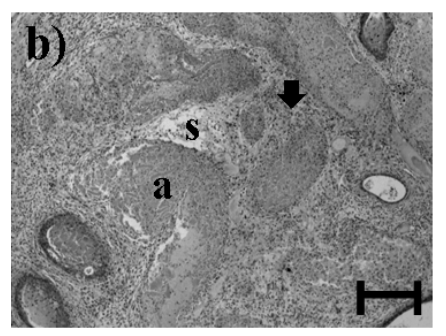

5 days post-lesion

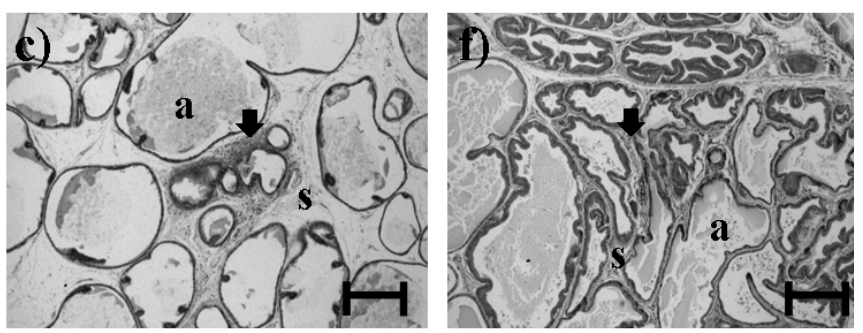

20 days post-lesion

Figure 3: Histological sections taken from dorsal (left panels) and ventral (right panels) prostatic lobes before and after chemical lesion. a, d) Before lesion the acinus and stroma show their normal morphological characteristics, with no leukocyte infiltration. b, e) Prostatic tissue 5 days after chemical lesion shows the presence of macrophages and sparse neutrophyles in the stroma, indicating an inflammatory process. c, f) The inflammatory process is diminished in the prostatic tissue at day 20 after lesion. (arrow=inflammatory process; slices stained with hematoxiline-eosine; $\mathrm{Bar}=200 \mu \mathrm{m}$ ). acinus (a); stroma (s)
In order to address the possibility that the lack of an effect of chemically-lesioning the ventral prostatic lobes could have been due to insufficient damage to this tissue, we did a second experiment in which the ventral prostatic lobes were completely removed surgically. No behavioral effects of ventral lobe ablation were observed either at 5 or 20 days post-surgery. Moreover, this procedure did not alter any seminal fluid or seminal plug parameters. Thus, pre-lesion data were similar to those shown in Tables I and II, respectively, and no changes in any seminal fluid or seminal plug parameter were observed at 5 or 20 days post-ablation (data not shown).

Experiment 3. Assessment of fertility after chemically lesioning the dorsal prostatic lobes or ablation of the ventral prostatic lobes

Figure 4 shows the percentage of females that became pregnant in different time periods (1-5, 6-10 and 11-15 days) of a 15-day test period. Most females became pregnant within the first 5 days of cohabitation with unlesioned males. However, 20 days after chemically lesioning their dorsal prostatic lobes,

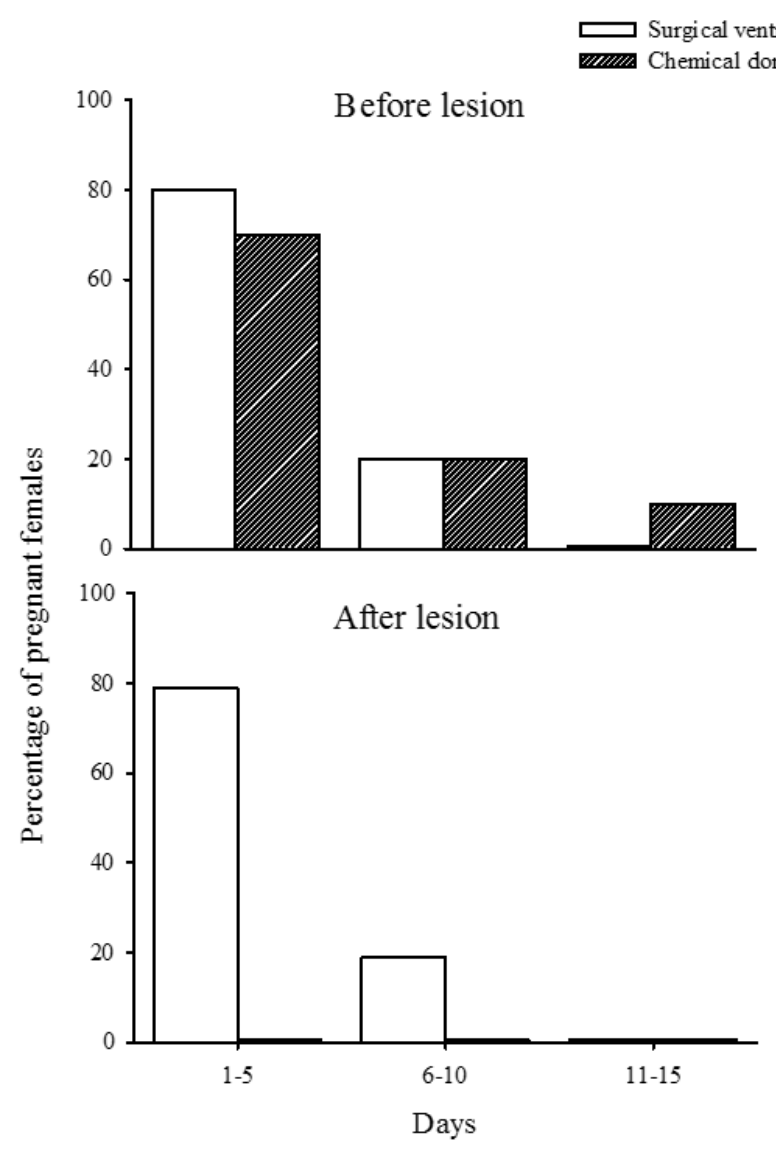

Figure 4: Percentage of females that became pregnant after cohabitation with males before (upper panel) and after (lower panel) they received a chemical lesion of dorsal prostate lobes (white bars) or surgical ablation of ventral prostate lobes (diagonal lines bars). Induction of pregnancy was identified at different periods (1-5, 6-10 and 11-15 days). 
cohabitation with these same males did not result in a single pregnancy across the 15 day test period (Fig. 4a). In striking contrast, ablation of the ventral lobes had no effect of fertility (Fig. 4b).

\section{DISCUSSION}

The rat prostate comprises dorsal, ventral, and lateral lobes. In the present study, we focused on the participation of the dorsal and ventral prostatic lobes in the production of ejaculate and their importance for fertility. Dorsal or ventral prostatic lobes were chemically or surgically lesioned, and the resulting changes in the characteristics of the seminal fluid, seminal plug, and fertility were examined. Lesions of the lateral prostatic lobes were not performed due to their close anatomical association with the major pelvic ganglia, which innervate penis erectile tissue, bulbourethral glands, and other reproductive structures (Hebel and Stromberg, 1986; Dail et al., 1989). Thus, lesioning the lateral prostatic lobes would result in collateral damage to these nervous ganglia, producing a failure of penile erection and preventing the display of intromission and ejaculation motor patterns. In fact, the removal of the dorsolateral prostatic lobes resulted in complete infertility (Queen et al., 1981).

Our findings are novel relative to those of Queen et al. (1981) in several important respects. In our study, the damage due to injection of the sclerosing agent into the dorsal or ventral prostatic lobes was restricted to the injected lobe; no histological damage was observed in the non-injected adjacent prostatic lobe or in the adjacent tissues. In this way, we were able to test the unique contribution of the dorsal prostatic lobe without damaging the lateral one; by contrast, in the study of Queen et al. (1981), both dorsal and lateral lobes were removed. Moreover, in our study the effect of dorsal or ventral prostate damage on copula, ejaculate and fertility was evaluated at 5 and 20 days post-lesion, whereas in the Queen et al. (1981) study only fertility was analyzed. Thus, we found that neither dorsal nor ventral prostatic lobe affected copulatory parameters. Notably, in the Queen et al. study, the authors did not register copulatory parameters of the male, therefore, they were not able to distinguish whether reduced fertility was due to negative effects of the ablation on copulatory behavior or ejaculate quality.

Lesioning the dorsal prostatic lobes produced two major effects on the ejaculate. First, the adhesion of the seminal plug to the vaginal walls was prevented. Although seminal plugs were coagulated and maintained their weight, size, and volume, their wet surface, consisting of seminal fluid containing motile spermatozoa, prevented their vaginal adhesion. Therefore, sperm transport was affected, probably due to a lack of hydrostatic pressure exerted by the seminal plug, which normally pushes spermatozoa from the vagina through the cervix, to the uterine horns (Blandau, 1945). As a consequence, the uterine horns contained only uterine fluid, for that reason, the samples collected showed a value of zero for viscosity. Nevertheless, it has been described in intact males, that viscosity depends on the secretions from the accessory sexual glands (Blandau, 1945).

Previous studies have shown that ablations of different portions of the seminal vesicles affect seminal plug weight: when a greater amount of tissue is excised, a lighter seminal plug is obtained (Carballada and Esponda, 1992). Removal of seminal vesicles also produced infertility in male rats (Queen et al. 1981). Moreover, it was demonstrated that when coagulating glands were totally removed, no seminal plug formed after coitus, and sperm transport to the uterus did not occur, therefore, males were completely sterile (Carballada and Esponda, 1992). In intact males, once the seminal plug is firmly lodged into vagina, many spermatozoa reach the uterine horns (Matthews Jr and Adler, 1978).

In other studies, reduced fertility has also been associated with an improperly positioned seminal plug. Male rats treated with finasteride, a 5 alfa-reductase inhibitor, show reductions in the weight of both seminal vesicles and prostate, and deposit small and inadequately positioned seminal plugs, resulting in reduced fertility (Cukierski et al., 1991).

Our results indicate that adhesion of the seminal plug to the vaginal walls is critical for sperm transport, and this adhesion requires secretions of the dorsal prostatic lobes. Using histochemical markers, it has been demonstrated that dorsal prostate secretes glycoconjugates. Dorsal lobes are rich in Man, GlcNac, Fuc, Gal/GalNac and oligosaccharides (Chan and Ho, 1999). Furthermore, dorsal prostate secretes dorsal-proteins I and II (Seitz et al., 1990). Dorsal-protein I is a major protein of the dorsal prostate (Kinbara and Cunha, 1996), and, compared to dorsal-protein I, dorsal-protein II has higher carbohydrate content (Wilson and French, 1980). The role of dorsal-proteins has not yet been established, but they apparently do not have an enzymatic function, since they are at a high concentration in cytosol (Wilson and French, 1980). It remains to be determined whether some of these secretions of the dorsal prostatic lobes promote seminal plug adhesion to the vaginal walls. In the present study, because sperm transport was disturbed by lesioning the dorsal lobes, sperm concentration in the uterine horns was also altered. At 5 days post-lesion, no spermatozoa were found in the uterus, and at 20 days post-lesion, sperm concentration (Austin and Dewsbury, 1986) was dramatically lower compared to that obtained from the same males before surgery, as well as compared to that reported previously (Lucio et al., 2009).

The second effect of dorsal prostatic lobe lesions was on sperm motility in the uterus. At day 20 post-lesion, when spermatic transport occurred, more than $40 \%$ of spermatozoa presented in situ motility versus $53 \%$ of spermatozoa with rapid progressive motility in intact males. This decrease in sperm motility was unexpected, because sperm motility is typically associated with zinc, which is secreted mainly by the lateral prostatic lobes (Lin et al., 2000). Immotile spermatozoa are stored in the cauda epididymis. Sperm motility is induced when spermatozoa are in contact with secretions from the accessory sex glands at ejaculation (Lindholmer, 1974). The prostate produces proteins giving an adequate medium for the survival of sperm, and enhances their motility in the female reproductive tract (Chow and $O, 1998)$. Human semen includes semenogelin; this protein inhibits sperm progressive motility, nevertheless, prostate-specific antigen (PSA) hydrolyses semenogelin, resulting in sperm motility (Robert and Gagnon, 1996). Patients having low sperm mobility also showed low PSA levels (Ahlgren et al., 1995). Therefore, it is possible that secretions of the rat dorsal prostate include hydrolytic enzymes that, when released into the seminal fluid, facilitate sperm motility through the female reproductive tract. In fact, it has been described that the vertebrate prostate, among another glands, produces glycosidases, exopeptidasas, and 
phospholipases. These enzymes digest proteins in the seminal plasma and on the surface of the spermatozoa, resulting in the facilitation of sperm motility (Vanha-Perttula et al., 1990).

In addition to the aforementioned enzymes, other factors are known to promote sperm motility. Fructose, secreted mainly by the dorsal prostate and coagulating glands, is reported to be a source of energy for the motility of spermatozoa (Mann, 1964). Since coagulating glands were intact in our experimental males, it seems that the presence of zinc from lateral prostate and the amount of fructose secreted by coagulating glands are not sufficient, and that additional components secreted by the dorsal prostatic lobes are necessary, for normal sperm motility. Besides fructose, prostatic secretion contains high concentrations of monovalent and divalent cations such as $\mathrm{Na}, \mathrm{K}, \mathrm{Zn}, \mathrm{Ca}$ and $\mathrm{Mg}$, as well as citric acid and many enzymes (Wilson et al., 1993). The interaction of some of these secretions and ions secreted by the dorsal prostate may affect the metabolism and function of spermatozoa. Considering the low sperm concentration and the reduced percentage of spermatozoa with rapid progressive motility, it was not surprising that all males with dorsal prostatic lesions were infertile.

We do not know exactly why the ventral prostatic lobe suffered less damage from the sclerosing agent, but it is possible that differences in tissue morphology between the dorsal and ventral lobes could have influenced the inflammatory response to this compound. The acini of the dorsal lobes are quite large and less convoluted than either the ventral or lateral lobes and are loosely distributed within the stromal tissue, which consists of an assortment of tissue elements that include extracellular material, small nerve endings, blood vessels, fibromuscular material, and fibroblasts. The acini are lined mainly with cuboidal cells, the secretions of which stain with an intensity between that of the lateral and ventral lobes (Jesik et al., 1982). These morphological characteristics could be related to the intensity of the inflammatory response of the prostatic lobes. In humans, for example the peripheral zone of the prostate is the most susceptible to inflammation and is where the majority of carcinomas occur versus the central zone, which is more resistant (Cunha et al., 1987). It has been described that the dorsal and lateral prostatic lobes in the rat are equivalent to the prostate peripheral zone in man (Price, 1963). Tissue damage caused by inflammation could impair prostatic function, perhaps causing a decreased secretion of the kallikrein-like proteolytic enzyme and an increase in the $\mathrm{pH}$ of semen, resulting in inadequate medium for sperm physiology (Motrich et al., 2009). In our study, the poor motility shown by the sperm from dorsal prostatic lesioned males could be another cause for their lack of fertility.

In contrast to the dramatic effects of dorsal lobe lesions, chemical lesion or ablation of the ventral prostate did not change the characteristics of the semen or seminal plug. In agreement with the results obtained by Queen et al. (1981), we found that removal of ventral lobes had no effect on the latency to become pregnant or on the percentage of females that became pregnant, indicating that these prostatic lobes do not play a critical role in reproductive parameters of male rats.

Thus, the present results indicate that, in the male rat, dorsal prostatic lobes are crucial for reproductive success, whereas the ventral lobes apparently are not necessary for reproduction, or participate only marginally. This is the first study in which the parameters of semen and seminal plug were evaluated using a simple method involving semen acquired from naturally inseminated females. It will be necessary to focus on dorsal prostatic lobe to determine the biochemical components that allow the adhesion of seminal plug to vaginal walls and also its participation in sperm motility.

\section{ACKNOWLEDGMENTS}

This work was partially supported by Consejo Nacional de Ciencia y Tecnología (CONACYT 105502 to RAL). JLTL (188574) and AALG (198782) were fellows from CONACYT.

\section{REFERENCES}

AHLGREN G, RANNEVIK G, LILJA H (1995) Impaired secretory function of the prostate in men with oligo-asthenozoospermia. J Androl 16:491-498.

AUSTIN D, DEWSBURY D (1986) Reproductive capacity of male laboratory rats. Physiol Behav 37:627-632.

BLANDAU RJ (1945) On the factors involved in sperm transport through the cervix uteri of the albino rat. Am J Anat 77:253-272.

CARBALLADA R, ESPONDA P (1992) Role of fluid from seminal vesicles and coagulating glands in sperm transport into the uterus and fertility in rats. J Reprod Fertil 95:639-648.

CHAN FL, HO SM (1999) Comparative study of glycoconjugates of the rat prostatic lobes by lectin histochemistry. Prostate 38:1-16.

CHOW PH, O WS (1998) Effects of male accessory sex glands on sperm transport, fertilization and embryonic loss in golden hamsters. Int J Androl 12:155-163.

CUKIERSKI MA, SINA JL, PRAHALADA S, WISE LD, ANTONELLO JM, MACDONALD JS, ROBERTSON RT (1991) Decreased fertility in male rats administered the 5 alfa-reductase inhibitor, finasteride, is due to deficits in copulatory plug formation. Reprod Toxicol 5:353-362.

CUNHA GR, DONJACOUR AA, COOKE PS, MEE S, BIGSBY RM, HIGGINS SJ, SUGIMURA Y (1987) The endocrinology and developmental biology of the prostate. Endocrinol Rev 8:338-362.

DAIL WG, TRUJILLO D, DE LA ROSA D, WALTON G (1989) Autonomic innervation of reproductive organs: analysis of the neurons whose axons project in the main penile nerve in the pelvic plexus of the rat. Anat Rec 224:94-101.

GUNN SA, GOULD TC (1957) A correlative anatomical and functional study of the dorsolateral prostate of the rat. Anat Rec 128:41-53.

GRIFFIN DJ, OKE EJ, CHO KJ, GIKAS PW (1986) Chemical ablation of the canine kidney using sodium tretradecyl sulphate (Sotradecol). A histopathologic study. Invest Radiol 21:217-220.

HAYASHI N, SUGIMURA Y, KAWAMURA J, DONJACOUR AA, CUNHA GR (1991) Morphological and functional heterogeneity in the rat prostatic gland. Biol Reprod 45:308-321.

HEBEL R, STROMBERG MW (1986) Anatomy and Embriology of the Laboratory Rat. Germany: BioMed Verlag Wörthree. pp:1-271.

HUMASON GL (1972) Animal Tissue Techniques. San Francisco: WH Freeman and Co. pp:1-641.

HUMPHREY GF, MANN T (1949) Studies on the metabolism of semen; citric acid in semen. Biochem J 44:97-105.

JESIK CJ, HOLLAND JM, LEE C (1982) An anatomic and histology study of the rat prostate. Prostate 3:81-97.

KIMBARA H, CUNHA GR (1996) Ductal heterogeneity in rat dorsal-lateral prostate. Prostate 28:58-64.

LAWS AND CODES OF MÉXICO (1995) Seventh Title. The Regulations of the General Law of Health Research. México. 12 $2^{\text {th }}$ updated Porrúa ed. pp:430-431.

LINDHOLMER C (1974) The importance of seminal plasma for human sperm motility. Biol Reprod 10:533-542.

LIN YC, CHANG TC, TSENG YJ, LIN YL, HUANG FJ, KUNG FT, CHANG SY (2000) Seminal plasma zinc levels and sperm motion characteristics in infertile samples. Chang Gung Med J 23:260-266.

LUCIO RA, FLORES-ROJAS G, AGUILAR F, ZEMPOALTECA R, PACHECO P, VELÁZQUEZ-MOCTEZUMA J (2001) Effects of genitofemoral nerve transection on copulatory behavior and fertility in male rats. Physiol Behav 73:487-492.

LUCIO RA, TLACHI JL, LÓPEZ AA, ZEMPOALTECA R, VELÁZQUEZMOCTZUMA J (2009) Analysis of the parameters of the ejaculate in the laboratory Wistar rat: technical description. Vet Mex 4:205-215. 
LUKE MC, COFFEY DS (1994) The male sex accessory tissues. KNOBIL E, NELLY JD (eds) In The Physiology of Reproduction. Raven Press: New York. pp:1435-1489.

MANN T (1964) The Biochemistry of Semen and of the Male Reproductive Tract. Methuen, London. New York. pp:493.

MANZO J, VÁZQUEZ MI, CRUZ MR, HERNÁNDEZ ME, CARRILLO P, PACHECO P (2000) Fertility ratio in male rats: Effects after denervation of two pelvic floor muscles. Physiol Behav 68:611-618.

MATTHEWS M, ADLER NT (1977) Facilitative and inhibitory influences of reproductive behavior on sperm transport in rats. J Comp Physiol Psychol 91:727-741.

MATTHEWS MK Jr, ADLER NT (1978) Systematic interrelationship of mating, vaginal plug position, and sperm transport in the rat. Physiol Behav 20:303-309.

MEISEL RL, SACHS BD (1994) The physiology of male sexual behaviour. KNOBIL E, NELLY JD (eds) In The Physiology of Reproduction. Raven Press: New York. Vol 2 pp:3-106.

MOTRICH RD, MACKERN-OBERTI JP, MACCIONI M, RIVERO V (2009) Effects of autoimmunity to the prostate on the fertility of the male rat. Fertil Steril 91:2273-2280.

PRESNELL JK, SCHREIBMAN MP (1997) Animal Techniques. Baltimore The Johns Hopkins University Press. pp:1-572.

PRICE D, WILLIAMS-ASHMAN HG (1961) The accessory reproductive glands of mammals. YOUNG ED WC (ed) In Sex and Internal Secretions. Baltimore Williams \& Wilkins Press. pp: 366-448.

PRICE D (1963) Comparative aspects of development and structure in the prostate. Natl Cancer Inst Monogr 12:1-27.
QUEEN K, DHABUWALA CB, PIERREPOINT CG (1981) The effect of the removal of the various accessory sex glands on the fertility of male rats. J Reprod Fertil 62:423-426.

ROBERT M, GAGNON C (1996) Purification and characterization of the active precursor of a human sperm motility inhibitor secreted by the seminal vesicles: identity with semenogelin. Biol Reprod 55:813-821.

SETCHELL BP, MADDOCKS S, BROOKS DE (1994) Anatomy, vasculature, inervation and fluids of the male reproductive tract. KNOBIL E, NELLY JD (eds) In The Physiology of Reproduction. Raven Press: New York. Vol 1 pp:1063-1177.

SEITZ J，KEPPLER C，RAUSCH U，AUMULLER G (1990) Inmunohistochemistry of secretory transglutaminase from rodent prostate. Histochemistry 93:525-530.

VANHA-PERTTULA T, JAUHIAINEN A (1983) Aminopeptidases of the rat prostatic complex and seminal vesicles: secretion and effect of castration. Mol Cell Endocrinol 32:195-204.

VANHA-PERTTULA T, RONKKO S, LAHTINEN R (1990) Hydrolases from bovine seminal vesicle, prostate and Cowper's gland. Andrologia 22:10-24.

WILSON MJ, GARCIA B, WOODSLON M, SINHA AA (1993) Gelatinolytic and caseinolytic proteinase activities in the secretions of the ventral, lateral and dorsal lobes of the rat prostate. Biol Reprod 48:1174-1184.

WILSON EM, FRENCH FS (1980) Biochemical homology between rat dorsal prostate and coagulating gland. Purification of a major androgeninduced protein. J Biol Chem 25:10946-109WILSON MJ, SINHA AA, POWELL JEH, ESTENSEN RD (1988) Plasminogen activator activities in the developing rat prostate. Biol Reprod 38:723-731.

ZAR JH (1999) Biostatistical Analysis. Prentice-Hall Inc. pp:1-663. 
